\title{
Continuous renal replacement therapy to reduce inflammation in a piglet hemorrhage-reperfusion extracorporeal membrane oxygenation model
}

\author{
Thornton S. Mu', Eldon G. Palmer', Sherreen G. Batts' ${ }^{1}$, Sarah L. Lentz-Kapua', Jane H. Uyehara-Lock² \\ and Catherine F.T. Uyehara ${ }^{3}$
}

BACKGROUND: During extracorporeal membrane oxygenation (ECMO), circulation of blood across synthetic surfaces triggers an inflammatory response. Therefore, we evaluated the ability of continuous renal replacement therapy (CRRT) to remove cytokines and reduce the inflammatory response in a piglet hemorrhage-reperfusion ECMO model.

METHODS: Three groups were studied: (i) uninjured controls ( $n=11$ ); (ii) hemorrhage-reperfusion while on venoarterial ECMO (30\% hemorrhage with subsequent blood volume replacement within $60 \mathrm{~min})(n=8)$; (iii) treatment with CRRT after hemorrhage-reperfusion while on ECMO $(n=7)$. Hemodynamic parameters, oxygen utilization, and plasma and broncho-alveolar lavage (BAL) cytokine levels were recorded and lung tissue samples collected for histologic comparison.

RESULTS: Whereas mean arterial pressures decreased among hemorrhage-reperfusion piglets, ECMO with CRRT did not significantly alter mean arterial pressures or systemic vascular resistance and was able to maintain blood flow as well as oxygen delivery after hemorrhage-reperfusion. Plasma interleukin (IL)- 6 and IL-10, and BAL tumor necrosis factor (TNF)- $\alpha$, IL-1 $\beta$, IL-6, IL-8, and IL-10 increased as a result of hemorrhage-reperfusion while on ECMO. After a 6-h period of CRRT, plasma IL-6 and BAL TNF-a, IL-6, and IL-8 levels decreased.

CONCLUSION: Data suggest CRRT may decrease inflammatory cytokine levels during the initial phase of ECMO therapy following hemorrhage-reperfusion while maintaining cardiac output and oxygen utilization.

$E^{x}$ tracorporeal membrane oxygenation (ECMO) is used to support patients with cardiac and/or respiratory failure that is unresponsive to conventional therapies. A large body of data exists that demonstrates its usefulness in neonatal and pediatric patients in cases of unresponsive pulmonary hypertension, respiratory failure, sepsis, and emergency temporary cardiac support $(1,2)$.

However, direct circulation of blood across synthetic surfaces escalates a pro-inflammatory response, further exacerbating a disease process that is already associated with the activation of the inflammatory cascade (3). In addition, the lung plays a major role as a site of inflammation during extracorporeal therapy (4). Levels of pro-inflammatory cytokines, such as tumor necrosis factor (TNF)- $\alpha$, interleukin (IL)- $1 \beta$, IL- 6 , and IL-8, are increased in patients as early as $3-4 \mathrm{~h}$ after placement on ECMO and/or cardiopulmonary bypass (CPB) (4-8).

In addition to exacerbation of the inflammatory cascade, ECMO therapy is associated with volume overload, acute renal failure, and blood loss from coagulopathy and consumption $(9,10)$. Management of these fluid imbalances either with crystalloid, colloid, or blood products can still paradoxically produce a progressive destruction of reversibly damaged cells. This "reperfusion injury" has a multifactorial etiology but appears to be strongly associated with an inflammatory response. With the return of blood flow, several inflammatory processes may occur that potentiate ischemic injury, including the following: leukocyte adhesion and infiltration, free radical release, and production of pro-inflammatory cytokine molecules (11). Therefore, improved therapeutic approaches that can diminish the inflammatory response secondary to pro-inflammatory cytokines are needed.

One such strategy involves continuous renal replacement therapy (CRRT). CRRT operates via several pathways of inflammatory mediator elimination, to include adsorption, diffusion, and convection (12-14). Although CRRT has been reported to demonstrate efficacy in the removal of IL-6 in adults with septic shock (15), its use in neonatal and pediatric ECMO populations was initially reported in the setting of concurrent acute renal failure $(9,16)$, Recently, some have suggested expanding the role of CRRT into non-renal settings, such as ECMO, CPB, and septic shock, for its "blood cleansing" abilities $(1,12)$.

The goal of this research project was to investigate whether removal of cytokines via CRRT can improve lung function and decrease inflammation in a piglet hemorrhage-reperfusion ECMO model. We hypothesized that removal of pro-inflammatory cytokines will reduce the overall inflammatory response and improve hemodynamic function and oxygen utilization. 


\section{RESULTS}

\section{Hemodynamics and Oxygen Utilization}

Among ECMO/hemorrhage-reperfusion piglets that did not receive CRRT (group 2), mean arterial pressures decreased with hemorrhage $(47 \pm 5 \mathrm{~mm} \mathrm{Hg})$ and remained significantly lower in group 2 than timed controls (group 1) at $\mathrm{S}+6 \mathrm{~h}(65$ $\pm 6 \mathrm{~mm} \mathrm{Hg}$ ) even with blood volume replacement. CRRT piglets' (group 3) mean arterial pressures $(77 \pm 4 \mathrm{~mm} \mathrm{Hg})$ were comparable with levels in timed controls at $\mathrm{S}+6 \mathrm{~h}(P<0.05)$ (Figure 1a). ECMO was able to maintain blood flow after hemorrhage-reperfusion, with no significant differences in cardiac output among all piglets at $\mathrm{S}+6 \mathrm{~h}$. (Figure $1 \mathrm{~b}$ )

Neither oxygen delivery nor oxygen consumption differed significantly among hemorrhage-reperfusion or CRRT piglets as compared with timed controls at $\mathrm{S}+6 \mathrm{~h}$. (Figure 2a,b) In order to maintain steady-state oxygen consumption needs, oxygen extraction ratios were consistently increased among hemorrhage piglets $(0.55 \pm 0.07)$, but as consumption demands decreased among CRRT piglets, their oxygen extraction ratios decreased $(0.45 \pm 0.07)$ to levels comparable with timed controls $(P<0.05)$ (Figure $2 \mathrm{c})$.

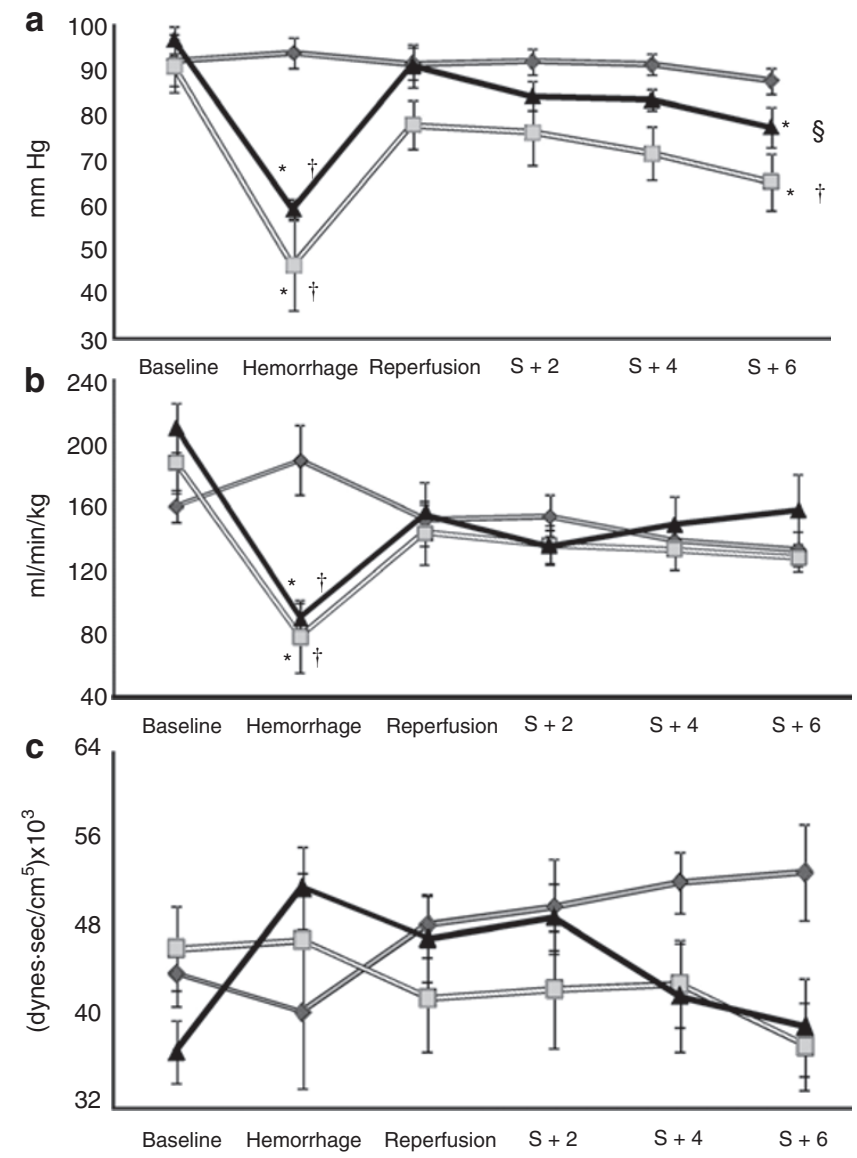

Figure 1. Hemodynamic parameters among treatment groups. (a) Mean arterial pressure; (b) cardiac output; (c) systemic vascular resistance. Baseline = baseline period; hemorrhage; reperfusion; $S+n=$ steady state $+n \mathrm{~h} .{ }^{*}=$ Significantly different from baseline; $\dagger=$ significantly different from timed controls; $\S=$ significantly different from hemorrhage $(P<0.05)$. Dark gray diamond = group 1, control; light gray square = group 2 , hemorrhage; black triangle = group 3 , continuous renal replacement therapy.
There were no significant differences in ventilator settings, i.e., peak inspiratory pressure, rate, or peak end expiratory pressure, required to maintain targeted tidal volumes of $6-8 \mathrm{ml} / \mathrm{kg}$.

\section{Cytokine Analyses}

No fluctuations in cytokine levels were observed during ECMO before hemorrhage, or after hemorrhage/before reperfusion.

Among hemorrhage-reperfused piglets, plasma IL-6 increased from baseline $27.2 \pm 14.2 \mathrm{pg} / \mathrm{ml}$ to $202.2 \pm 110.8 \mathrm{pg} /$ $\mathrm{ml}$ at $\mathrm{S}+6$ as a result of hemorrhage-reperfusion while on ECMO $(P<0.05)$ (Figure 3a). Similarly, plasma IL-10 concentration increased from $9.8 \pm 6.9 \mathrm{pg} / \mathrm{ml}$ to $28.7 \pm 20.8 \mathrm{pg} / \mathrm{ml}$ at $\mathrm{S}+6$ while on ECMO $(P<0.05)$ (Figure $3 \mathrm{~b})$.

After a 6 -h period of CRRT, plasma IL-6 concentrations $(63.1 \pm 19.0 \mathrm{pg} / \mathrm{ml})$ decreased $(P<0.05)$ (Figure 3a). However, plasma IL-10 concentrations remained relatively constant (Figure $3 \mathrm{~b}$ ). The majority of piglets in each treatment group had undetectable plasma TNF- $\alpha$ concentrations, and consequently, no significant changes were noted. Similarly, IL-1 $\beta$ and IL-8 concentrations were essentially undetectable in plasma among piglets across the treatment groups but did appear to
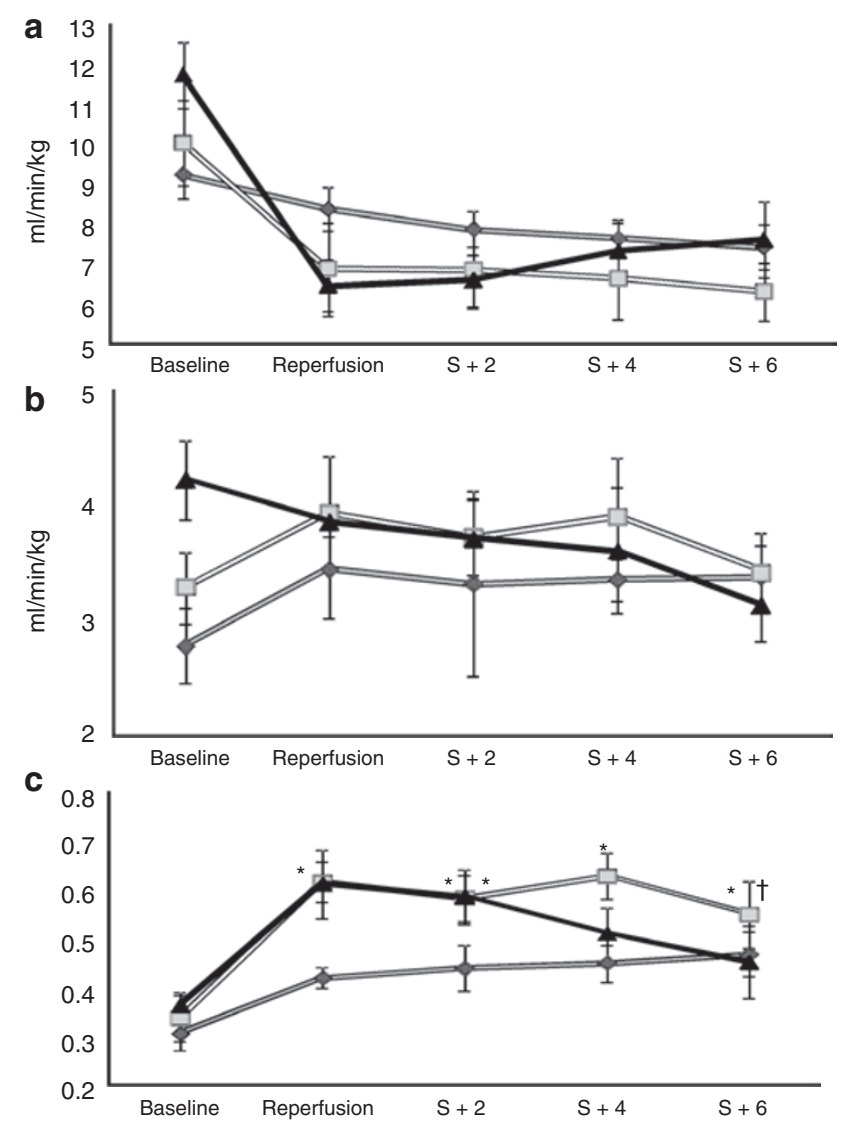

Figure 2. Oxygen utilization among treatment groups. (a) Oxygen delivery; (b) oxygen consumption; (c) oxygen extraction. Baseline = baseline period; reperfusion; $\mathrm{S}+n=$ steady state $+n \mathrm{~h} .{ }^{*}=$ Significantly different from baseline; $\dagger=$ significantly different from timed controls $(P<0.05)$. Dark gray diamond = group 1, control; light gray square = group 2, hemorrhage; black triangle = group 3 , continuous renal replacement therapy. 
be preferentially increased in tissue broncho-alveolar lavage (BAL) specimens.

BAL TNF- $\alpha(157.0 \pm 43.9 \mathrm{pg} / \mathrm{ml}), \mathrm{IL}-1 \beta(1410.6 \pm 417.0 \mathrm{pg} /$ $\mathrm{ml})$, IL-6 (151.5 $\pm 40.0 \mathrm{pg} / \mathrm{ml}), \mathrm{IL}-8(14591.0 \pm 4005.1 \mathrm{pg} / \mathrm{ml})$, and IL-10 $(40.9 \pm 26.0 \mathrm{pg} / \mathrm{ml})$ levels increased as a result of hemorrhage-reperfusion while on ECMO at $S+6(P<0.05)$ (Figure 4a-e).

After a 6-h period of CRRT, BAL TNF- $\alpha(36.0 \pm 12.6 \mathrm{pg} /$ $\mathrm{ml})$, IL-6 $(43.4 \pm 8.9 \mathrm{pg} / \mathrm{ml})$, and IL-8 $(3059.2 \pm 1219.8 \mathrm{pg} /$ $\mathrm{ml})$ levels decreased $(P<0.05)$. IL-1 $\beta(482.1 \pm 218.7 \mathrm{pg} / \mathrm{ml})$ concentrations showed a tendency toward decline, whereas IL-10 (1.4 $\pm 0.8 \mathrm{pg} / \mathrm{ml})$ levels remained relatively unchanged. Lastly, although nonbronchoscopic BAL appears well tolerated, significant increases in BAL IL-1 $\beta$ (1484.7 $\pm 600.2 \mathrm{pg} /$ $\mathrm{ml})$ and IL-8 $(7341.3 \pm 2587.8 \mathrm{pg} / \mathrm{ml})$ levels were noted over the 6-h steady state period among timed controls $(P<0.05)$ (Figure 4a-e).

\section{Tissue Analyses}

There were no significant differences in the histologic analyses among the three groups of piglets. The average score for timed controls was $1.17 \pm 0.61$ as compared with $1.54 \pm 0.54$ for hemorrhage-reperfusion piglets and $1.47 \pm 0.67$ for CRRT piglets. The most common finding observed was the presence of intra-alveolar polymorphonuclear lymphocytic infiltrates (Figure 5).
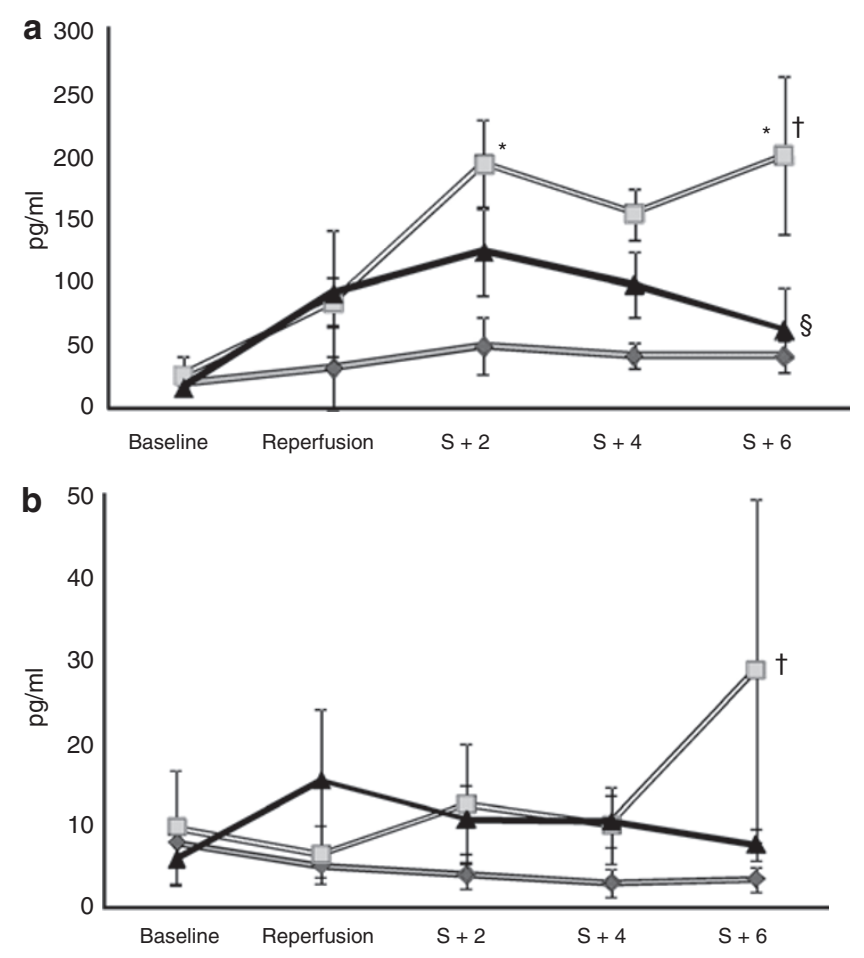

Figure 3. Plasma cytokine concentrations among treatment groups. (a) Interleukin (IL)- 6 and (b) IL-10. Baseline = baseline period; reperfusion; $\mathrm{S}+n=$ steady state $+n \mathrm{~h} .{ }^{*}=$ Significantly different from baseline; $\dagger=$ significantly different from timed controls; $\S=$ significantly different from hemorrhage $(P<0.05)$. Dark gray diamond = group 1, control; light gray square = group 2 , hemorrhage; black triangle $=$ group 3 , continuous renal replacement therapy.

\section{DISCUSSION}

To date, our findings are the first in vivo animal trial in an ECMO model to demonstrate that CRRT lowers inflammatory cytokine concentrations without altering hemodynamic function and oxygen utilization. Previous studies have cited the important role that pro-inflammatory cytokines play in the extracorporeal circulation setting. Marti et al. (8) demonstrated that at as early as $6 \mathrm{~h}$, serum concentrations of IL- $1 \beta$, IL-6, and TNF- $\alpha$ are elevated in patients on CPB, implicating their role as important mediators of acute inflammatory response from exposure of blood components to synthetic surfaces. We suspect the differences between patient populations (human vs. porcine) as well as the underlying disease processes influenced the differences in IL- $1 \beta$ and TNF- $\alpha$ levels in our study. Furthermore, Golej et al. (6) demonstrated that IL- $1 \beta$ and IL- 8 appear to be preferentially increased in BAL fluid as compared with serum samples during a 5 -h period in a piglet ECMO model. In addition, they observed increases in the anti-inflammatory cytokine IL-10 in BAL fluid. As in our study, serum IL-1 $\beta$ levels were essentially undetectable. Although their findings implicated the role of the pulmonary system in inflammatory mediator production, they did not make any correlation with clinical well-being, i.e., worsening hemodynamic or oxygen utilization (6). Utilizing the same cytokine markers, we were able to make similar conclusions in our experimental ECMO model. In addition, we were able to distinguish CRRT hemofiltration as a means of mitigating the increased inflammatory response and correlate it with clinical improvements.

Despite the differences in age, size, and underlying comorbidities, adult CRRT findings have been extrapolated to pediatric populations because the underlying mechanisms of solute clearance are the same, as well as the utilization of weight-based operational parameters. Although adsorption appears to be the predominant mechanism by which cytokines are initially cleared from the circulation, this mechanism reaches a saturation limit within a few hours. Therefore, CRRT's additional mechanisms of action, namely, convection (hemofiltration) and, to a lesser extent, dialysis, likely provide additional cytokine clearance (12-14). Pediatric CRRT, as well as neonatal CRRT with ECMO, have been limited by case reports, small sample size, and single-center studies, making generalization and standardization of practice difficult across pediatric patients and centers $(17,18)$. Additional reviews of institutional experiences with CRRT and ECMO described the technical challenges and complications associated with continuous venovenous hemofiltration $(\mathrm{CVVH})$ and ECMO using objective clinical and/or biochemical markers $(9,16)$. Recently, Paden et al. (19), suggested that the combination of CVVH and ECMO does not contribute to development of chronic renal failure but is associated with higher mortality risk than ECMO alone, probably because of the preexisting renal failure rather than the introduction of CVVH. In addition, Santiago et al. (20) analyzed the efficacy and safety of the inclusion of CRRT with ECMO in vitro, in a piglet model, as well as in selected pediatric patients. Although the use of 

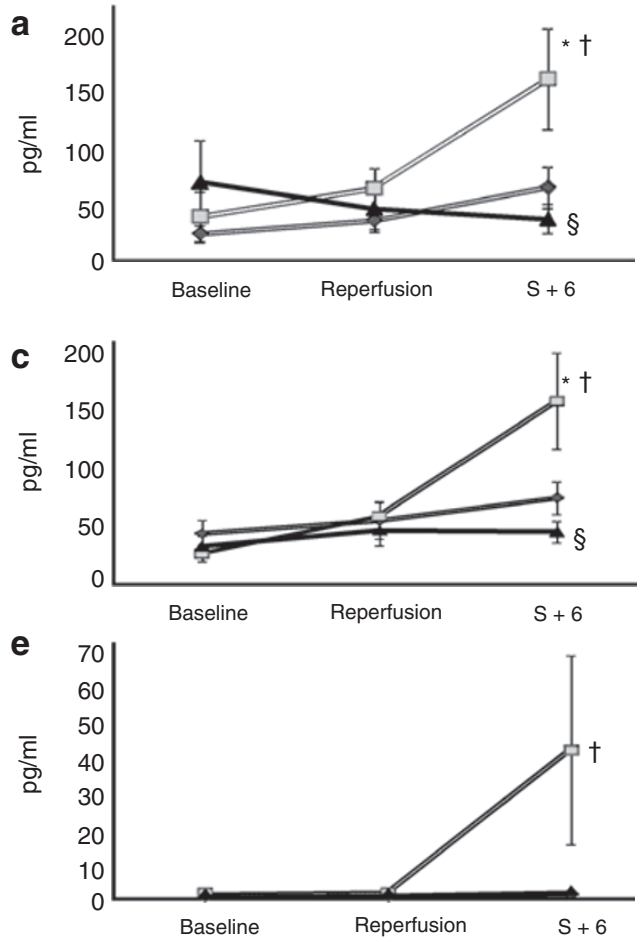
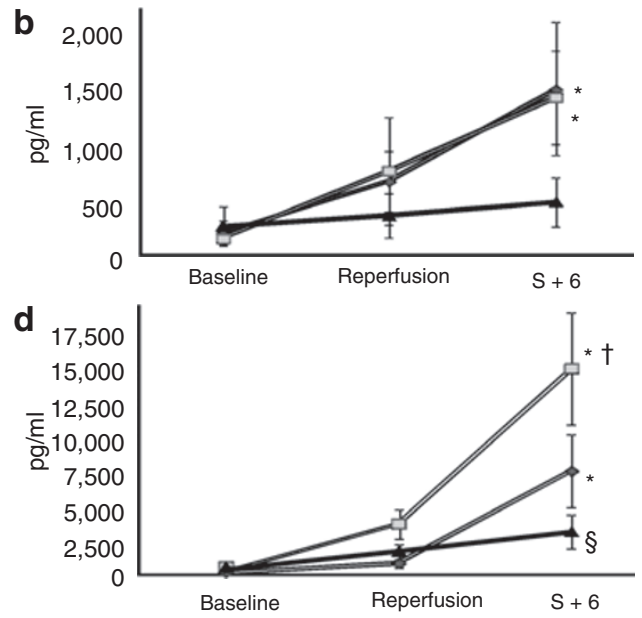

Figure 4. Broncho-alveolar lavage cytokine concentrations among treatment groups. (a) Tumor necrosis factor-a, (b) interleukin (IL)-1 $\beta$, (c) IL-6, (d) IL-8, and (e) IL-10. Baseline = baseline period; reperfusion; $\mathrm{S}+n=$ steady state $+n \mathrm{~h}$. ${ }^{*}=$ Significantly different from baseline; $†=$ significantly different from timed controls; $\S=$ significantly different from hemorrhage $(P<0.05)$. Dark gray diamond $=$ group 1 , control; light gray square $=$ group 2 , hemorrhage; black triangle $=$ group 3 , continuous renal replacement therapy.

CRRT was noted to be safe and effective for fluid balance control, they did not discuss its potential for cytokine removal. Therefore, the aim of our study was to evaluate the efficacy of CRRT in reducing cytokine levels while improving hemodynamics and oxygen utilization in a piglet ECMO model of hemorrhage-reperfusion.

In a trial of early continuous venovenous hemodiafiltration (CVVHDF)in a piglet model of endotoxin-induced acute lung injury without ECMO, Lu et al. (21) reported improved cardiopulmonary function, decreased serum inflammatory cytokine levels (TNF- $\alpha$ and soluble TNF receptor), and improved pulmonary function in the CVVHDF group during a 6-h-long steady-state period. An in vitro study using pooled human blood investigating continuous hemofiltration during ECMO demonstrated that plasma IL-6, IL-8, and IL-1 receptor antagonist concentrations were significantly lower, whereas no significant influence of hemofiltration was observed for TNF- $\alpha$ and IL-1 $\beta$ levels 22 . Although our study was not able to conclusively correlate improved hemodynamic profile with decreased pro-inflammatory cytokine levels, we broadened the number of observed cytokine markers that increased with injury and subsequently declined with CRRT and also differentiated between tissue (BAL) vs. plasma cytokine origin.

The effect of the anti-inflammatory cytokine IL-10 on the CRRT piglets is unclear; the hemofilter operates on the molecular weight of the molecules and therefore is unable to differentiate between pro- and anti-inflammatory cytokines. What may be more clinically relevant is the observation of relatively unchanged IL-10 concentrations on CRRT that can continue to exert their anti-inflammatory properties which are beyond the scope of our 6-h period of observation.

Interestingly, our study clearly demonstrated a difference in cytokine levels in the circulation vs. BAL, indicating differential regulation of cytokine responses from lung tissue sources and other sources contributing to blood levels. The preferential BAL cytokine expression reported in our trial is similar to a report of greater expression of TNF- $\alpha$, IL-6, and IL-8 from alveolar leukocytes than plasma leukocytes in humans on $\mathrm{CPB}$ for $\sim 2 \mathrm{~h}$. Of the cytokines studied, IL- 8 gene expression was greater in lung tissue than plasma, reflecting the degree of pulmonary dysfunction (23). In contrast, a 24-h in vitro ECMO run using pooled human blood demonstrated elevations in plasma IL- 6 and IL- 8 levels, but by design no BAL/tissue specimens were available for comparison (22).

The use of non-bronchoscopic BAL for treatment of meconium aspiration syndrome to improve oxygenation with a targeted volume of $10-15 \mathrm{ml} / \mathrm{kg}$ diluted surfactant saline suspension has been reported with modest success in both animal and human models $(24,25)$. Non-bronchoscopic BAL has also been reported in ECMO patients on lung rest who are not dependent on native pulmonary function for gas exchange (26). We chose to use this technique to allow the comparison of plasma vs. tissue sources of cytokine production. Although our data could be confounded by inherent lung injury from the BAL procedure itself, we accounted for this by establishing a baseline with timed controls to compare cytokine levels with the two other piglet groups. In addition, we used dilute surfactant in the BAL to mitigate the effect of saline-induced 


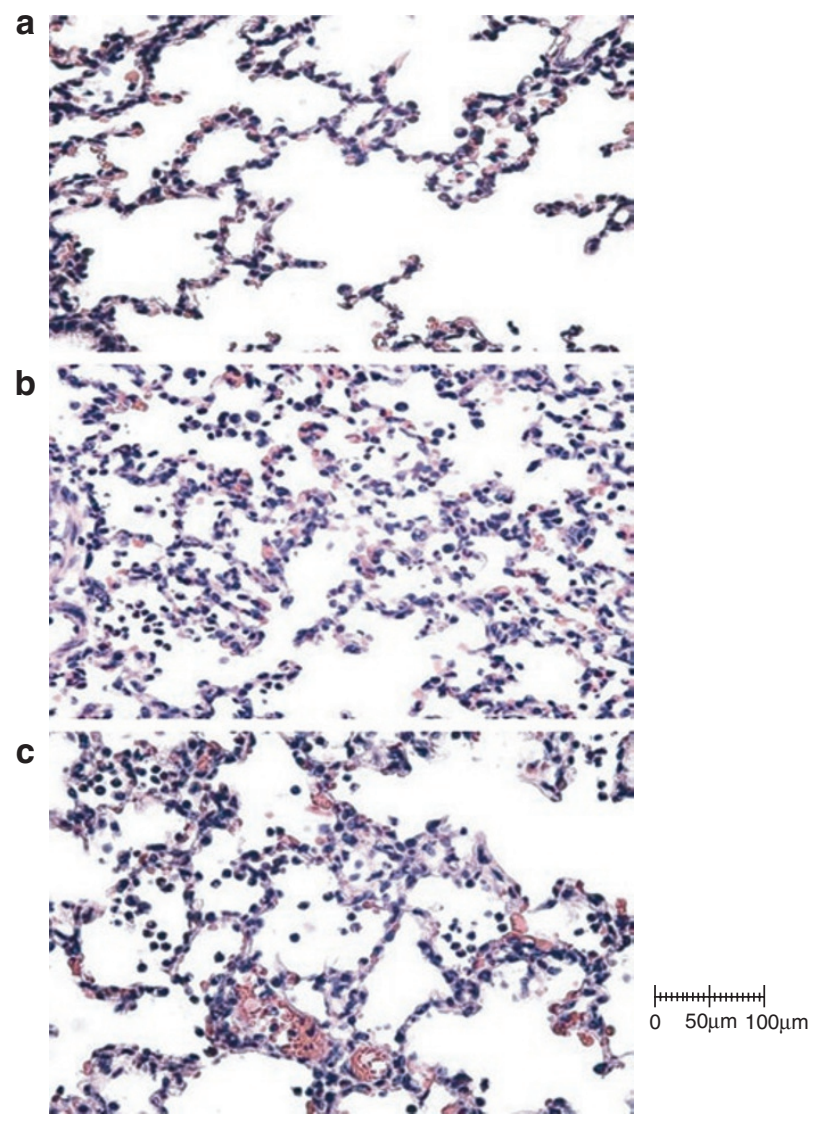

Figure 5. Histological review of lung specimens. (a) Uninjured timed control with no intra-alveolar inflammatory cells. (b) Hemorrhagereperfusion piglet demonstrating moderate presence of intra-alveolar inflammatory cells. (c) Continuous renal replacement therapy piglet with slightly decreased number of inflammatory cells within the alveolar space. Original magnification $\times 400(\mathbf{a}-\mathbf{c})$. removal of endogenous surfactant production following a modified protocol (27).

The lack of significant histologic differences among our experimental arms may be the result of a number of causes. Modest differences among the histologic scores may be a reflection of less severe lung injury related to the limited study duration or the result of ECMO as "lung rest/protection" modality. Therefore, detecting significantly different scores would require a much larger sample size beyond the scope of our experimental protocol.

There are some limitations to our study. We did not include an additional control group of piglets on ECMO without hemorrhage-reperfusion because the focus of our study was to determine the efficacy of cytokine clearance in an animal model with established cytokine upregulation associated with an underlying disease/injury state. As discussed in the results, analyses obtained shortly after ECMO initiation and before hemorrhage-reperfusion did not produce substantially elevated cytokine levels, and therefore we designed our model to combine the cumulative effect of ECMO and hemorrhage-reperfusion in order to establish substantial cytokine upregulation. Bradykinin release syndrome is a well-known response when blood comes into contact with AN69 membranes used in CRRT circuits, which can contribute to our inflammatory cytokine profiles (28). We mitigated this effect by continuously monitoring $\mathrm{pH}$ in the ECMO circuit and administering additional sodium bicarbonate after CRRT initiation if acidosis was observed. Similar to other studies, our experimental timeline spanned only a 6 -h steady state period, and may limit its applicability in the clinical realm, where ECMO therapy lasts for days to weeks. Furthermore, the limited study duration may not detect cytokine elevations that peak later, such as IL-1 $\beta$, whose peak may occur as late

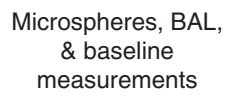

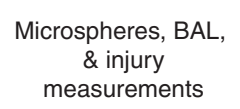

measurements Microspheres
\& steady state
measurements

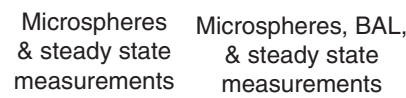
measurements measurements

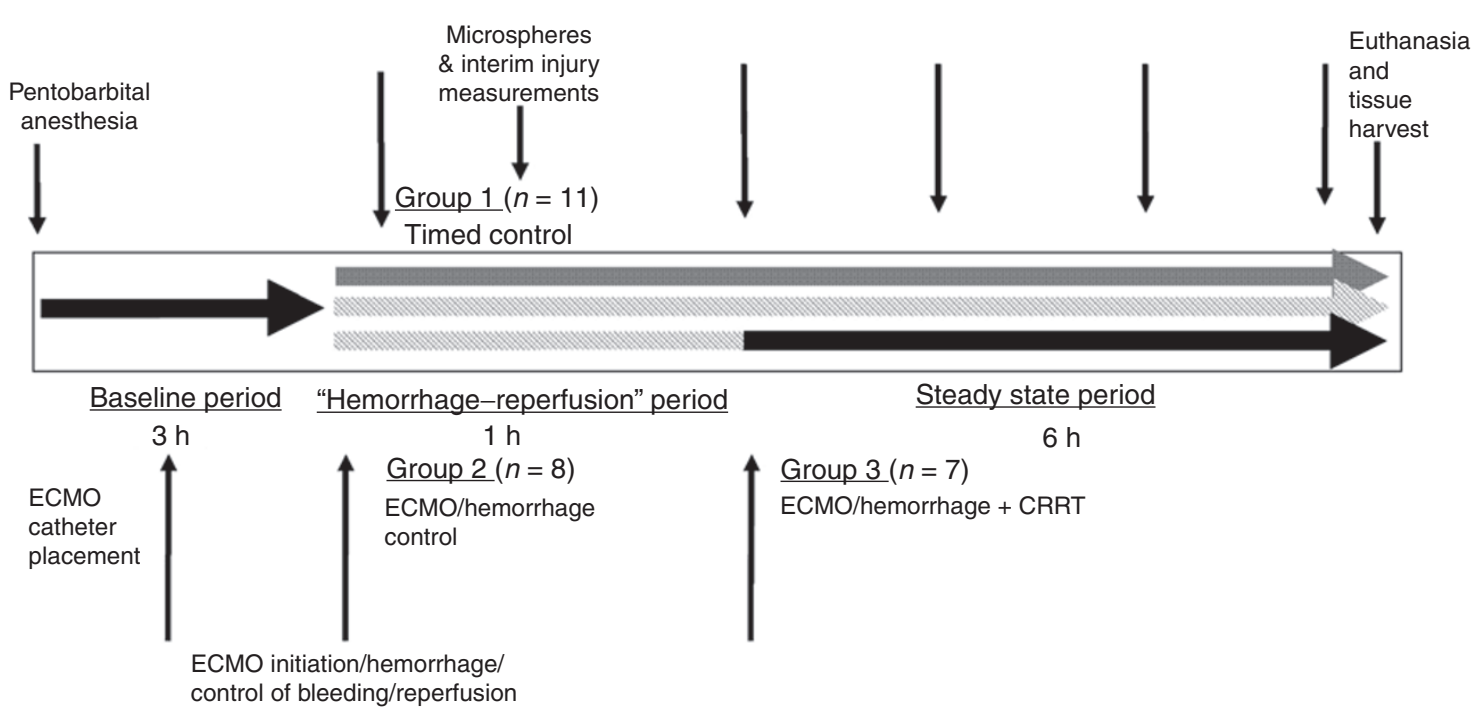

Figure 6. Experimental protocol timeline. BAL, broncho-alveolar lavage; CRRT, continuous renal replacement therapy; ECMO, extracorporeal membrane oxygenation. Dark gray = group 1, control; light gray = group 2, hemorrhage; black = group 3, CRRT. 
as $24 \mathrm{~h}$ following $\mathrm{CPB}$ (7). Our focus therefore only addresses the effect of removal of cytokine elevations that occur during the period following ECMO initiation. The effect of longterm consequences of this strategy on hemodynamics and oxygen utilization warrants further study.

In conclusion, placement on venoarterial ECMO with subsequent hemorrhage-reperfusion increases inflammatory cytokines in both plasma and BAL specimens and has a detrimental effect on overall hemodynamic function and oxygen utilization in our experimental model. There appears to be preferential expression of certain cytokines from lung tissue (BAL) vs. plasma specimens. CRRT may decrease inflammatory cytokine levels during the initial phase of ECMO therapy following hemorrhage-reperfusion while maintaining cardiac output and oxygen utilization.

\section{METHODS}

This study was approved by the Institutional Animal Care and Use Committee at Tripler Army Medical Center. Investigators complied with the policies as prescribed in the National Research Council's Guide for the Care and Use of Laboratory Animals and the USDA Animal Welfare Act. Animals were handled in accordance with the National Institutes of Health guidelines in facilities fully accredited by the American Association for Accreditation of Laboratory Animal Care International.

\section{Animal Preparation}

Sus scrofa Yorkshire-cross piglets of either gender weighing 7-9kg were sedated initially with intraperitoneal pentobarbital $30 \mathrm{mg} / \mathrm{kg}$. A surgical plane of anesthesia was maintained using additional doses $(5-30 \mathrm{mg} /$ $\mathrm{kg}$ ) of pentobarbital every $1 \mathrm{~h}$ or more frequently as needed. Piglets were intubated with a $5.0 \mathrm{~mm}$ cuffed endotracheal tube and mechanically ventilated in a pressure-controlled mode (Maquet Servo-I, Solna, Sweden) with fraction of inspired oxygen $\left(\mathrm{FiO}_{2}\right)=21 \%$, inspiratory time $=0.35 \mathrm{~s}$, respiratory rate $=40 \mathrm{bpm}$, peak end expiratory pressure $=2 \mathrm{~cm} \mathrm{H}_{2} \mathrm{O}$, peak inspiratory pressure $=12 \mathrm{~cm} \mathrm{H}_{2} \mathrm{O}$. Peak inspiratory pressure was titrated to maintain a tidal volume of $6-8 \mathrm{ml} / \mathrm{kg}$ and rate was adjusted to maintain end-tidal $\mathrm{CO}_{2}$ of $35-45$ torr $(4.7-6.0 \mathrm{kPa})$. Continuous mean arterial blood pressure monitoring and cardiac output calculations by thermodilution were obtained via a 5-6 French catheter situated in the abdominal aorta. A 5-6 French Swan-Ganz catheter was placed via the left femoral vein for continuous pulmonary artery pressure, pulmonary artery wedge pressure, continuous infusion of normal saline at $0.1 \mathrm{ml} / \mathrm{kg} / \mathrm{min}$ to maintain euhydration, medication administration, and venous blood sampling. Final position was verified by characteristic pressure wave form.

Central venous pressure, blood pressures, and urine output were continuously monitored and used as an indication of appropriate resuscitation.

All pressure monitoring catheters were coupled to transducers, zeroed to the level of the mid-heart of the pigletand connected to a cardiorespiratory monitor (Philips MP70, Boeblingen, Germany). Standard cardiovascular function and blood oxygenation calculations were performed for the calculation of cardiac output, heart rate, stroke volume, systemic vascular resistance, pulmonary vascular resistance, oxygen content, oxygen delivery, oxygen extraction, and shunt fraction. Measurements are reported as mean values \pm SEM.

\section{Experimental Protocol}

Piglets were divided into three groups: group 1 uninjured timed controls $(n=11)$, group $2 \mathrm{ECMO} /$ hemorrhage-reperfusion controls $(n=$ 8 ), and group $3 \mathrm{ECMO} /$ hemorrhage-reperfusion treated with CRRT $(n=7)$ (Figure 6).

After the initial 60 -min period of stabilization after catheterization, piglets were monitored for a 20 -min period defined as the baseline before the initiation of venoarterial ECMO in groups 2 and 3. Hemodynamic measurements and blood gases were obtained at baseline, during and after hemorrhage-reperfusion injury on ECMO, and at three time points $(S+2, S+4, S+6 h)$ during the 6-h steadystate period after reperfusion. Group 1 timed controls were monitored at the same corresponding time points as in groups 2 and 3.

\section{Blood Samples}

Along with the usual hemodynamic measurements and blood gases, arterial blood samples were drawn for hormone, electrolyte, and cytokine analyses. Control animals in group 1 were sampled at the same time points of hemorrhage-reperfusion periods as group 2 and 3 animals. After separation of the plasma, the red cells were returned to the piglet after reconstitution with equivalent volume of normal saline to maintain blood volume and oxygenation.

\section{Extracorporeal Circuit}

ECMO cannulae were placed for venoarterial ECMO delivery with a 8-10 French cannula placed in the aortic arch via the left internal carotid artery, a 12-14 French venous cannula aligned with the right atrium via the right external jugular vein, and an 8-10 French cephalad cannula via the right external jugular vein for additional venous flow. ECMO circuits were primed by the introduction of $\mathrm{CO}_{2}, 0.9 \%$ saline solution, and then filled with whole blood freshly harvested within $24 \mathrm{~h}$ from donor Sus scrofa pigs weighing $50 \mathrm{~kg}$ (donor pigs were anesthesized with isoflurane and bled via a left internal carotid artery catheter cut-down). Sodium bicarbonate, calcium chloride, and 100 units of heparin were then added. The total priming volume was $450 \mathrm{ml}$. ECMO flow was driven by centrifugal pump (Jostra-Rotaflow HL20, Maquet, Rastatt, Germany) through a hollow-fiber membrane oxygenator (Quadrox, Jostra, Maquet, Rastatt, Germany) After cannulation, peak end expiratory pressure and inspiratory time were increased to improve functional residual capacity. $\mathrm{FiO}_{2}$ remained at $21 \%$, respiratory rate halved, and peak inspiratory pressure adjusted to maintain a tidal volume of $6-8 \mathrm{ml} / \mathrm{kg}$ as part of a "lung rest" strategy on ECMO.

\section{Hemorrhage-Reperfusion Model}

To further induce an inflammatory response in groups 2 and 3, additional blood volume was removed from the ECMO circuit to create an $\sim 50 \%$ decrease in mean arterial blood pressure. Approximately $15 \mathrm{ml} / \mathrm{kg}$ of whole blood, or $30 \%$ of blood volume of the piglet, was removed over a $15-$ min period. Thirty minutes of stabilization followed to simulate "control of bleeding" and then blood volume was transfused to target a return to baseline mean arterial pressures to simulate "reperfusion."

\section{CRRT}

CVVHDF was performed using the Prismaflex machine consisting of four roller pumps with air detector, bubble trap, pressure limiter (Gambro, Lakewood, CO), and a hemofilter (M60 membrane filter, Gambro), an AN69 membrane with a cutoff point of $<30,000 \mathrm{Da}$ and surface area of $0.6 \mathrm{~m}^{2}$. The AN69 is an acrylonitrile and sodium methallyl sulfonate high-flux membrane with an adsorptive function usually saturated within a few hours. Total saline priming volume of $93 \mathrm{ml}$ was utilized. The inlet (arterial) and outlet (venous) lines of the CRRT were connected via separate three-way valves before the centrifugal pump. Zero-balanced CVVHDF was initiated after completion of hemorrhage-reperfusion time period and continued for the 6-h steady-state period. CVVHDF was performed with a blood flow rate of $75 \mathrm{ml} / \mathrm{min}(\sim 9-10 \mathrm{ml} / \mathrm{kg} / \mathrm{min})$, dialysis rate of $500 \mathrm{ml} / \mathrm{h}$ with PrismaSate BGK 4/0/1.2 (containing bicarbonate, glucose, potassium, magnesium with an osmolarity of $296 \mathrm{mOsm} / \mathrm{l}$; Gambro), post-filter ultrafiltration rate of $50 \mathrm{ml} / \mathrm{h}$ with normal saline, and prefilter replacement rate of $350 \mathrm{ml} / \mathrm{h}(\sim 35 \mathrm{ml} / \mathrm{kg} / \mathrm{h})$ with PrismaSate BGK 4/0/1.2.

\section{Protocol for BAL}

Non-bronchoscopic BAL was performed using a volume of $16 \mathrm{ml} /$ $\mathrm{kg}$ diluted surfactant saline suspension ( $2 \mathrm{mg}$ surfactant $/ \mathrm{ml}$ fluid) at the following three time points: baseline, after injury (ECMO/hemorrhage-reperfusion), and at the end of steady state $(S+6 h)$. The entire procedure lasted $\sim 2 \mathrm{~min}$ and has been previously described (27). Porcine lipid extract surfactant was used (Curosurf, Chiesi 
Farmaceutici SpA, Parma, Italy). After collection, the samples were centrifuged at $4,000 \mathrm{rpm}$ for $15 \mathrm{~min}$, and the supernatant and pellet were separated and frozen at $-70^{\circ} \mathrm{C}$ for further analyses.

\section{Cytokine Assays}

Plasma and BAL supernatant concentrations of circulating proinflammatory cytokines TNF- $\alpha$, IL- $1 \beta$, IL- 6 , and IL- 8 and the antiinflammatory cytokine marker IL-10 were measured using commercially available solid-phase enzyme-linked immunosorbent assay kits specific for swine cytokines (ALPCO Diagnostics, Salem, NH) using monoclonal antibodies specific for each test marker. All samples and standards were assayed in duplicate, and inflammatory protein concentrations were determined on the basis of a standard curve and reported as mean values \pm SEM.

\section{Tissue Sampling}

All animals were killed after the last observed treatment period. Tissue samples from the upper, middle, and lower segments of each lobe of the lung were collected from the piglets and fixed in $10 \%$ buffered formalin. Six-micrometer-thick paraffin sections were stained with hematoxylin and eosin. These sections were examined under a light microscope by a pathologist blinded to the treatment groups. A four-point $(0,1,2$, and 3$)$ scoring system was used to assess individual characteristics of each section for intra-alveolar inflammatory cells (other than macrophages), alveolar fibrin edema, septal thickening, and alveolar hemorrhage by both extent and severity. These variables were scored using this scale: $0=$ no injury, $1=$ mild injury $(<25 \%)$, $2=$ moderate injury $(25-50 \%), 3=$ severe injury $(>50 \%)$. A total score was calculated from the sum of the individual scores.

\section{Statistical Analyses}

Values are expressed as means \pm SEM. Data were analyzed using a two-way ANOVA with repeated measures over time (JMP 4.0.4 program, SAS Institute, Cary, NC). Post hoc comparisons between treatment groups were performed with Student's $t$-test. A $P$ value of $<0.05$ was considered significant.

\section{ACKNOWLEDGMENTS}

We thank Aileen Sato, Claudia Hernandez, Wayne Ichimura, January Andaya, Lee-Ann Murata, Glenn Hashiro, Solomon Nair, Tabitha Smith, Matt Ursery, Norman Kreiselmeier, Joseph Anderson, Jacob Espinosa, and Michael Lustik for their tireless support and dedication to this project.

The views expressed in this article are those of the authors and do not reflect the official policy or position of the Department of the Army, Department of Defense, or the US government.

\section{STATEMENT OF FINANCIAL SUPPORT}

This work was supported, in part, by the US Army Medical Research and Material Command Core Research Program, FY08 War Supplemental Intramural Research Program, and Congressionally Directed Medical Research Program grant W23RYX6200N602.

\section{REFERENCES}

1. Fortenberry JD, Paden ML. Extracorporeal therapies in the treatment of sepsis: experience and promise. Semin Pediatr Infect Dis 2006;17:72-9.

2. Ford JW. Neonatal ECMO: current controversies and trends. Neonatal Netw 2006;25:229-38.

3. Fortenberry JD, Bhardwaj V, Niemer P, Cornish JD, Wright JA, Bland L. Neutrophil and cytokine activation with neonatal extracorporeal membrane oxygenation. J Pediatr 1996;128(5 Pt 1):670-8.

4. Massoudy P, Zahler S, Becker BF, Braun SL, Barankay A, Meisner H. Evidence for inflammatory responses of the lungs during coronary artery bypass grafting with cardiopulmonary bypass. Chest 2001;119:31-6.

5. Mildner RJ, Taub N, Vyas JR, et al. Cytokine imbalance in infants receiving extracorporeal membrane oxygenation for respiratory failure. Biol Neonate 2005;88:321-7.

6. Golej J, Winter P, Schöffmann G, et al. Impact of extracorporeal membrane oxygenation modality on cytokine release during rescue from infant hypoxia. Shock 2003;20:110-5.
7. Adrian K, Mellgren K, Skogby M, Friberg LG, Mellgren G, Wadenvik H. Cytokine release during long-term extracorporeal circulation in an experimental model. Artif Organs 1998;22:859-63.

8. Martí F, Muñoz J, Peiró M, et al. Higher cytotoxic activity and increased levels of IL-1 beta, IL-6, and TNF-alpha in patients undergoing cardiopulmonary bypass. Am J Hematol 1995;49:237-9.

9. Cavagnaro F, Kattan J, Godoy L, et al. Continuous renal replacement therapy in neonates and young infants during extracorporeal membrane oxygenation. Int J Artif Organs 2007;30:220-6.

10. ECMO Registry of the Extracorporeal Life Support Organization (ELSO). Ann Arbor, Michigan 2010.

11. Rushing GD, Britt LD. Reperfusion injury after hemorrhage: a collective review. Ann Surg 2008;247:929-37.

12. Schetz M. Non-renal indications for continuous renal replacement therapy. Kidney Int Suppl 1999;56:88-94.

13. Matsuda K, Hirasawa H, Oda S, Shiga H, Nakanishi K. Current topics on cytokine removal technologies. Ther Apher 2001;5:306-14.

14. Prowle JR, Bellomo R. Continuous renal replacement therapy: recent advances and future research. Nat Rev Nephrol 2010;6:521-9.

15. Nakada TA, Oda S, Matsuda K, et al. Continuous hemodiafiltration with PMMA Hemofilter in the treatment of patients with septic shock. Mol Med 2008; 14:257-63

16. Shaheen IS, Harvey B, Watson AR, Pandya HC, Mayer A, Thomas D. Continuous venovenous hemofiltration with or without extracorporeal membrane oxygenation in children. Pediatr Crit Care Med 2007;8:362-5.

17. Symons JM, Chua AN, Somers MJ, et al. Demographic characteristics of pediatric continuous renal replacement therapy: a report of the prospective pediatric continuous renal replacement therapy registry. Clin J Am Soc Nephrol 2007;2:732-8.

18. Ricci Z, Polito A, Giorni C, Di Chiara L, Ronco C, Picardo S. Continuous hemofiltration dose calculation in a newborn patient with congenital heart disease and preoperative renal failure. Int J Artif Organs 2007;30:258-61.

19. Paden ML, Warshaw BL, Heard ML, Fortenberry JD. Recovery of renal function and survival after continuous renal replacement therapy during extracorporeal membrane oxygenation. Pediatr Crit Care Med 2011;12:153-8.

20. Santiago MJ, Sánchez A, López-Herce J, et al. The use of continuous renal replacement therapy in series with extracorporeal membrane oxygenation. Kidney Int 2009;76:1289-92.

21. Lu GP, Gong JY, Lu ZJ, Zhang LE, Kissoon N. Effect of continuous venovenous hemodiafiltration on endotoxin-induced acute lung injury of the piglets. Pediatr Crit Care Med 2011;12:e73-8.

22. Skogby M, Adrian K, Friberg LG, Mellgren G, Mellgren K. Influence of hemofiltration on plasma cytokine levels and platelet activation during extra corporeal membrane oxygenation. Scand Cardiovasc J 2000;34:315-20.

23. Kotani N, Hashimoto H, Sessler DI, et al. Cardiopulmonary bypass produces greater pulmonary than systemic proinflammatory cytokines. Anesth Analg 2000;90:1039-45.

24. Calkovska A, Mokra D, Drgova A, Zila I, Javorka K. Bronchoalveolar lavage with pulmonary surfactant/dextran mixture improves meconium clearance and lung functions in experimental meconium aspiration syndrome. Eur J Pediatr 2008;167:851-7.

25. Lista G, Bianchi S, Castoldi F, Fontana P, Cavigioli F. Bronchoalveolar lavage with diluted porcine surfactant in mechanically ventilated term infants with meconium aspiration syndrome. Clin Drug Investig 2006;26:13-9.

26. Mildner RJ, Taub NA, Vyas JR, et al. Repeatability of cellular constituents and cytokine concentration in fluid obtained by non-bronchoscopic bronchoalveolar lavage of infants receiving extracorporeal oxygenation. Thorax 2001;56:924-31.

27. Balaraman V, Meister J, Ku TL, et al. Lavage administration of dilute surfactants after acute lung injury in neonatal piglets. Am J Respir Crit Care Med 1998;158:12-7.

28. Brophy PD, Mottes TA, Kudelka TL, et al. AN-69 membrane reactions are pH-dependent and preventable. Am J Kidney Dis 2001;38:173-8. 